\title{
An unpublished theorem of Manfred Schocker and the Patras-Reutenauer algebra
}

\author{
Dieter Blessenohl
}

Received: 16 November 2007 / Accepted: 18 December 2007 / Published online: 24 January 2008

(C) Springer Science+Business Media, LLC 2008

\begin{abstract}
Patras, Reutenauer (J. Algebr. Comb. 16:301-314, 2002) describe a subalgebra $\mathfrak{A}$ of the Malvenuto-Reutenauer algebra $\mathcal{P}$. Their paper contains several characteristic properties of this subalgebra. In an unpublished manuscript Manfred Schocker states without proof a theorem, providing two further characterizations of the Patras-Reutenauer algebra. In this paper we establish a slightly generalized version of Schocker's theorem, and give some applications. Finally we describe a derivation of the convolution algebra $\mathfrak{A}$, which is a homomorphism for the inner product.
\end{abstract}

Keywords Symmetric group algebras · Reciprocity laws · Lie idempotents · Solomon's descent algebra

\section{Introduction}

In this section we explain some different characterizations of the Patras-Reutenauer algebra, contained in [9], and the theorem of Schocker.

As a vector space the Malvenuto-Reutenauer algebra $\mathcal{P}$ is the direct sum of all group algebras $K \mathcal{S}_{n}$, where $\mathcal{S}_{n}$ denotes the group of all permutations of the set $[n]:=\{1, \cdots, n\} \subseteq \mathbb{N}$. The set $\mathcal{S}:=\bigcup_{n \geq 0} \mathcal{S}_{n}$ is a basis of $\mathcal{P}$. The field of coefficients $K$ is assumed to be of characteristic 0 . Via Polya action $\mathcal{P}$ acts on every free associative algebra $\mathcal{A}=\mathcal{A}\langle X\rangle$, freely generated by a set $X$. The multiplicative monoid $X^{*}$ generated by $X$ is a basis of $\mathcal{A}$. For all $\sigma \in \mathcal{S}$ and all words $x_{1} \cdots x_{n} \in X^{*}$ of length $n$ we put

$$
\sigma x_{1} \cdots x_{n}:= \begin{cases}x_{1 \sigma} \cdots x_{n \sigma} & \text { if } \sigma \in \mathcal{S}_{n} \\ 0_{\mathcal{A}} & \text { if } \sigma \notin \mathcal{S}_{n}\end{cases}
$$

D. Blessenohl (凶)

Mathematisches Seminar, Christian-Albrechts-Universität Kiel, Ludewig-Meyn-Str. 4, 24098 Kiel, Germany

e-mail: blessenohl@math.uni-kiel.de 
Linear extension yields the Polya action of $\mathcal{P}$ on $\mathcal{A}$, which is a left action. For every subset $Y$ of $X$ we define a (uniquely determined) algebra endomorphism of $\mathcal{A}$ by

$$
x \mapsto x_{Y}:= \begin{cases}x & \text { for } x \in Y, \\ 1_{\mathcal{A}} & \text { for } x \in X \backslash Y .\end{cases}
$$

We write $a_{Y}$ for the image of $a$ under this mapping, for all $a \in \mathcal{A}$. The coproduct $\delta: \mathcal{A} \rightarrow \mathcal{A} \otimes \mathcal{A}$ is the uniquely determined algebra homomorphism, such that

$$
x \delta=x \otimes 1_{\mathcal{A}}+1_{\mathcal{A}} \otimes x,
$$

for all $x \in X$. The Lie subalgebra $\mathcal{L}:=\mathcal{L}\langle X\rangle$ generated by $X$ of the Lie algebra $\mathcal{A}_{\text {Lie }}$ associated to $\mathcal{A}$ is the set of all $a \in \mathcal{A}$ with the following property:

$$
a \delta=a \otimes 1_{\mathcal{A}}+1_{\mathcal{A}} \otimes a,
$$

by the theorem of Friedrichs. There is a coproduct $\downarrow$ on the vector space $\mathcal{P}$, too: for $\sigma \in \mathcal{S}_{n}$,

$$
\sigma \downarrow:=\sum_{j=0}^{n} \tau_{j} \otimes \rho_{n-j},
$$

$\tau_{j} \in \mathcal{S}_{j}$ and $\rho_{n-j} \in \mathcal{S}_{n-j}$, where, viewed as a word, the permutation $\tau_{j}$ is obtained by lining up the elements $1, \cdots, j$ from $\sigma$ in their present order. Similarly, the permutation $\rho_{n-j}$ is obtained by reading out the numbers $j+1, \cdots, n$ from $\sigma$ from left to right and subtracting $j$ from each of them. A more conceptual description is given in (3). For example, $312 \downarrow=312 \otimes \emptyset+1 \otimes 21+12 \otimes 1+\emptyset \otimes 312$. Another example is $1_{\mathcal{S}_{n} \downarrow} \downarrow \sum 1_{\mathcal{S}_{j}} \otimes 1_{\mathcal{S}_{n-j}}$. We embed $\mathcal{P}$ in $\mathcal{A}\langle\mathbb{N}\rangle$ simply by reading permutations as words over the alphabet $\mathbb{N}$. To be more precise, we define a linear mapping w of $\mathcal{P}$ into $\mathcal{A}\langle\mathbb{N}\rangle$ by

$$
\mathrm{w}: \sigma \mapsto \sigma \mathrm{w}:=1 \sigma . \cdots . n \sigma=\sigma(1.2 . \cdots . n) \in \mathbb{N}^{*},
$$

for all $\sigma \in \mathcal{S}_{n}$, and linear extension. ${ }^{1}$ By $*$ we denote the inner product on $\mathcal{P}$, inherited from the algebra structure of all $K \mathcal{S}_{n}$. Then,

$$
(x * y) \mathrm{w}=x(y \mathrm{w}),
$$

for all $x, y \in \mathcal{P}$. Finally put

$$
\mathcal{O}:=\{x \in \mathcal{P} \mid x \mathrm{w} \in \mathcal{L}\langle\mathbb{N}\rangle\}
$$

The elements of $\mathcal{O}$ are often called multilinear Lie elements. Obviously, $\mathcal{O}=$ $\bigoplus_{n \geq 1} \mathcal{O}_{n}$ where $\mathcal{O}_{n}:=\mathcal{O} \cap K \mathcal{S}_{n}$. In addition to the inner product the convolution product $\star$ on $\mathcal{P}$ is defined as follows: For all $\sigma \in \mathcal{S}_{n}$ and $\tau \in \mathcal{S}_{m}$ we define $\sigma \# \tau \in \mathcal{S}_{n+m}$ by

$$
i(\sigma \# \tau):=\left\{\begin{array}{clrl}
i \sigma & \text { for } & 1 & \leq i \leq n, \\
(i-n) \tau+n & \text { for } n+1 & \leq i \leq n+m,
\end{array}\right.
$$

\footnotetext{
${ }^{1}$ We denote the concatenation of $x, y \in \mathcal{A}\langle\mathbb{N}\rangle$ by $x . y$.
} 
then

$$
\sigma \star \tau:=\sum_{\rho}(\sigma \# \tau) \rho,
$$

where the summation is extended over all permutations $\rho \in \mathcal{S}_{n+m}$, which are increasing on $[n]$ and on $n+[m]=\{n+1, \cdots, n+m\}$. For example, $1 \star 21=$ $132+231+321$. This turns $\mathcal{P}$ into an associative algebra with neutral element $\emptyset$, the only element of $\mathcal{S}_{0}$. By $\mathfrak{A}$ we denote the unitary subalgebra of $(\mathcal{P}, \star)$ generated by $\mathcal{O}$. Observe $\mathfrak{A}=\bigoplus_{n>0} \mathfrak{A}_{n}$, where $\mathfrak{A}_{n}:=\mathfrak{A} \cap K \mathcal{S}_{n}$, i.e. $\mathfrak{A}$ is a homogeneous subspace of $\mathcal{P}$, just as $\mathcal{O}$. The first characterization of $\mathfrak{A}$ is a consequence of several statements in [9] (Proposition-Definition 3, Theorem 4 and the assertions about primitive elements):

Theorem 1.1 Let $x \in \mathcal{P}$. Then $x \in \mathfrak{A}$ if and only if for all sets $X$ and all $a \in \mathcal{A}:=$ $\mathcal{A}\langle X\rangle$ :

$$
(x a) \delta=(x \downarrow)(a \delta) .
$$

The left hand side refers to Polya action of $\mathcal{P}$ on $\mathcal{A}$ and the right hand side to Polya action of $\mathcal{P} \otimes \mathcal{P}$ on $\mathcal{A} \otimes \mathcal{A}$. In particular, $1_{\mathcal{S}_{n}} \in \mathfrak{A}$ for all $n \in \mathbb{N}$.

The second characterization needs some more preparation. Let $X$ be an alphabet and $<$ a total ordering on $X$. We define the standard permutation $\pi_{w} \in \mathcal{S}_{n}$ belonging to a word $w=x_{1} \cdots x_{n} \in X^{*}$ as follows:

$$
i \pi_{w}<j \pi_{w} \Longleftrightarrow\left\{\begin{array}{l}
x_{i}<x_{j}, \\
\text { or } x_{i}=x_{j} \text { and } i<j .
\end{array}\right.
$$

For example, let $a<b<\cdots<z$ and $w=$ rccacd, then $\pi_{w}=623145 \in \mathcal{S}_{6}$. By linear extension we get a mapping

$$
\text { st : } \mathcal{A}\langle X\rangle \rightarrow \mathcal{P}, \quad w \mapsto w s t:=\pi_{w} .
$$

Then the coproduct $\downarrow$ can be described as follows:

$$
\sigma \downarrow=\sum_{j=0}^{n}(\sigma \mathrm{W})_{[j]} \mathrm{st} \otimes(\sigma \mathrm{W})_{[n] \backslash[j]} \mathrm{st},
$$

for all $\sigma \in \mathcal{S}_{n}$. A slight modification of 1.1 follows:

Proposition 1.2 Let $x=\sum_{\sigma \in \mathcal{S}_{n}} k_{\sigma} \sigma \in K \mathcal{S}_{n}$. Then $x \in \mathfrak{A}$ if and only if

$$
(x \mathrm{w}) \delta=(x \downarrow)((1.2 . \cdots . n) \delta) .
$$

Proof By 1.1 the condition (4) is necessary since $x \mathrm{w}=x(1.2 . \cdots . n)$. On the other hand, if $X$ is a set and $a=x_{1} \cdots x_{n} \in X^{n}$ define the algebra homomorphism $\varphi$ : $\mathcal{A}\langle\mathbb{N}\rangle \rightarrow \mathcal{A}\langle X\rangle$ by

$$
i \varphi:= \begin{cases}x_{i} & \text { if } 1 \leq i \leq n \\ 1_{\mathcal{A}\langle X\rangle} & \text { if } n<i\end{cases}
$$


Then $\varphi$ is permutable with Polya action, i.e. $\left(\sigma i_{1} \ldots . i_{n}\right) \varphi=\sigma\left(i_{1} \varphi \cdots i_{n} \varphi\right)$, for all $\sigma \in \mathcal{S}_{n}$, further $x a=(x(1.2 . \cdots . n)) \varphi$ and

$$
\begin{aligned}
(x a) \delta & =(x(1.2 . \cdots . n)) \varphi \delta \\
& =(x(1.2 . \cdots . n)) \delta(\varphi \otimes \varphi) \\
& =((x \downarrow)(1.2 . \cdots . n) \delta)(\varphi \otimes \varphi) \\
& =(x \downarrow)((1.2 . \cdots . n) \delta(\varphi \otimes \varphi)) \\
& =(x \downarrow)(a \delta),
\end{aligned}
$$

therefore $x \in \mathfrak{A}$ by 1.1 .

Condition (4) is equivalent to

$$
\begin{aligned}
& \sum_{\substack{J \subseteq[n] \\
|J|=j}} \sum_{\sigma \in \mathcal{S}_{n}} k_{\sigma}\left((\sigma \mathrm{w})_{J} \otimes(\sigma \mathrm{w})_{[n] \backslash J}\right) \\
& \quad=\sum_{\substack{J \subseteq[n] \\
|J|=j}} \sum_{\sigma \in \mathcal{S}_{n}} k_{\sigma}\left((\sigma \mathrm{w})_{[j]} \operatorname{st}(1.2 . \cdots . n)_{J} \otimes(\sigma \mathrm{w})_{[n] \backslash[j]} \operatorname{st}(1.2 . \cdots . n)_{[n] \backslash J}\right),
\end{aligned}
$$

for $0 \leq j \leq n$. Applying st $\otimes$ st yields

$$
\begin{aligned}
& \sum_{\substack{J \subseteq[n] \\
|J|=j}} \sum_{\sigma \in \mathcal{S}_{n}} k_{\sigma}\left((\sigma \mathrm{w})_{J} \mathrm{st} \otimes(\sigma \mathrm{w})_{[n] \backslash J} \mathrm{st}\right) \\
&=\left(\begin{array}{c}
n \\
j
\end{array}\right) \sum_{\sigma \in \mathcal{S}_{n}} k_{\sigma}\left((\sigma \mathrm{w})_{[j]} \mathrm{st} \otimes(\sigma \mathrm{w})_{[n] \backslash[j]} \mathrm{st}\right) .
\end{aligned}
$$

The following combinatorial characterization of $\mathfrak{A}$ ([9], Theorem 4) is much stronger than this last assertion.

Theorem 1.3 Let $x=\sum_{\sigma \in \mathcal{S}_{n}} k_{\sigma} \sigma \in K \mathcal{S}_{n}$. Then $x \in \mathfrak{A}$ if and only if

$$
\begin{aligned}
& \sum_{\sigma \in \mathcal{S}_{n}} k_{\sigma}\left((\sigma \mathrm{w})_{J} \mathrm{st} \otimes(\sigma \mathrm{w})_{[n] \backslash J} \mathrm{st}\right) \\
&=\sum_{\sigma \in \mathcal{S}_{n}} k_{\sigma}\left((\sigma \mathrm{w})_{[|J|]} \mathrm{st} \otimes(\sigma \mathrm{w})_{[n] \backslash[|J|]} \mathrm{st}\right),
\end{aligned}
$$

for all $J \subseteq[n]$

The succeeding theorem of Manfred Schocker provides two further characterizations of the Patras-Reutenauer algebra. It is contained without proof in an unpublished manuscript.

Theorem 1.4 For all $x \in \mathcal{P}$ the following statements are equivalent: 
(i) $x \in \mathfrak{A}$,

(ii) $\left(z_{1} \star z_{2}\right) * x=\left(\left(z_{1} \otimes z_{2}\right) *_{\otimes} x \downarrow\right)$ conv, for all $z_{1}, z_{2} \in \mathcal{P}$,

(iii) $(x * z) \downarrow=x \downarrow *_{\otimes} z \downarrow$, for all $z \in \mathcal{P}$.

Here conv $: \mathcal{P} \otimes \mathcal{P} \rightarrow \mathcal{P}, x \otimes y \mapsto x \star y$ denotes the linearization of the convolution.

The statement (ii) is called the multiplicative reciprocity law. It was proved in [5] for elements $z_{1}, z_{2}, x$ in Solomon's algebra $\mathcal{D}$, which is the subalgebra of $(\mathcal{P}, \star)$ generated by all $1_{\mathcal{S}_{n}}$. In particular, $\mathcal{D} \subseteq \mathfrak{A}$. By [13] $\mathcal{D}$ is also a subalgebra of $(\mathcal{P}, *)$. In this paper we give a proof of Schocker's result in a slightly generalized version and some applications.

\section{The Lie projector $R$}

We present a useful instrument in this section, which is useful in the proof of Schocker's theorem and for applications.

Let $p_{n}$ be the canonical projection of $\mathcal{P}$ onto $K \mathcal{S}_{n}$. The algebra $(\mathcal{P}, \star)$ is graded since $K \mathcal{S}_{n} \star K \mathcal{S}_{m} \subseteq K \mathcal{S}_{n+m}$. We denote by

$$
\widehat{\mathcal{P}}:=\prod_{n \geq 0} K \mathcal{S}_{n}
$$

the completion of $\mathcal{P}$ with respect to the metric given by

$$
d(\alpha, \beta):=\left\{\begin{array}{cl}
e^{-\min \left\{n \mid p_{n}(\alpha-\beta) \neq 0\right\}} & \text { if } \alpha \neq \beta, \\
0 & \text { if } \alpha=\beta .
\end{array}\right.
$$

Consider $\mathcal{P}$ as a subspace of $\widehat{\mathcal{P}}$. The projection of $\widehat{\mathcal{P}}$ onto $K \mathcal{S}_{n}$ is again denoted by $p_{n}$. For every sequence $\left(\alpha_{n}\right)_{n \geq 0}$ converging to $0_{\widehat{\mathcal{P}}}$ the series $\sum \alpha_{n}$ itself is convergent. Cauchy multiplication turns $\widehat{\mathcal{P}}$ into an associative algebra with neutral element $\emptyset \in \mathcal{S}_{0}$. Obviously, $(\mathcal{P}, \star)$ is a subalgebra of $(\widehat{\mathcal{P}}, \star)$. The inner product $*$ and the Polya action of $\mathcal{P}$ on $\mathcal{A}$ can also be extended to $\widehat{\mathcal{P}}$. The latter is a homogeneous operation, i.e., the subspaces $\mathcal{A}_{n}$ are invariant, where $\mathcal{A}_{n}$ denotes the subspace of $\mathcal{A}$ generated by all elements of $X^{*}$ of length $n$. In contrast with $(\mathcal{P}, *)$ the algebra $(\widehat{\mathcal{P}}, *)$ has a neutral element:

$$
E:=\sum_{n \geq 0} 1_{\mathcal{S}_{n}}
$$

Polya action defines a linear mapping pol of $\widehat{\mathcal{P}}$ into the algebra $\mathcal{E}$ of all linear endomorphisms of $\mathcal{A}$, which is injective if (and only if) $X$ is infinite. With respect to the inner product pol is an anti-homomorphism. The following statement ${ }^{2}$ is well known:

$$
\operatorname{pol}(S \star T)=\delta(\operatorname{pol}(S) \otimes \operatorname{pol}(T)) \operatorname{conc}
$$

\footnotetext{
${ }^{2}$ In [7] this is used to define the convolution product on $\mathcal{P}$.
} 
for all $S, T \in \widehat{\mathcal{P}}$. Here conc $: \mathcal{A} \otimes \mathcal{A} \rightarrow \mathcal{A}, a \otimes b \mapsto a b$ denotes the linearization of the concatenation in $\mathcal{A}$. Putting

$$
\varphi \star \psi:=\delta(\varphi \otimes \psi) \text { conc }
$$

for all $\varphi, \psi \in \mathcal{E}$ turns $\mathcal{E}$ into an associative algebra with neutral element $\varepsilon$, the canonical projection of $\mathcal{A}=\bigoplus_{n \geq 0} \mathcal{A}_{n}$ onto $\mathcal{A}_{0}$. Then pol is a unital homomorphism of $(\widehat{\mathcal{P}}, \star)$ in $(\mathcal{E}, \star)$. It is convenient (see [11]), to generalize conc and $\delta$ in the following way: for all $k \in \mathbb{N}$ define an algebra homomorphism

$$
\delta^{(k)}: \mathcal{A} \rightarrow \underbrace{\mathcal{A} \otimes \cdots \otimes \mathcal{A}}_{k}=\mathcal{A}^{\otimes k}
$$

by

$$
\delta^{(k)}: x \mapsto \sum_{i=0}^{k-1} \underbrace{1_{\mathcal{A}} \otimes \cdots \otimes 1_{\mathcal{A}}}_{i} \otimes x \otimes \underbrace{1_{\mathcal{A}} \otimes \cdots \otimes 1_{\mathcal{A}}}_{k-i-1},
$$

for all $x \in X$, further $\left(a_{1} \otimes \cdots \otimes a_{k}\right)$ conc $^{(k)}:=a_{1} \cdots a_{k}$. Then for all $\varphi_{1}, \cdots, \varphi_{k} \in \mathcal{E}$

$$
\varphi_{1} \star \cdots \star \varphi_{k}=\delta^{(k)}\left(\varphi_{1} \otimes \cdots \otimes \varphi_{k}\right) \text { conc }^{(k)} \text {. }
$$

As a consequence of (6) and (5), observe the reciprocity law for Polya action:

$$
\left(S_{1} \star \cdots \star S_{k}\right) a=\left(\left(S_{1} \otimes \cdots \otimes S_{k}\right)\left(a \delta^{(k)}\right)\right) \operatorname{conc}^{(k)},
$$

for all $S_{1}, \cdots, S_{k} \in \widehat{\mathcal{P}}$ and all $a \in \mathcal{A}$. An element $P$ of $\widehat{\mathcal{P}}$ is a Lie projector, if $P * P=$ $P$ and if $P \mathcal{A}=\mathcal{L}$ for all sets $X$. As a consequence of (7), we note

$$
\left(P_{1} \star \cdots \star P_{k}\right) \mathcal{A} \subseteq \underbrace{\mathcal{L} \cdots \mathcal{L}}_{k}=: \mathcal{L}^{k},
$$

for all Lie projectors $P_{1}, \cdots, P_{k}$. Furthermore, (cf. [11], 1.5.6.)

$$
a \delta^{(k)}=\sum_{i=0}^{k-1} \underbrace{1_{\mathcal{A}} \otimes \cdots \otimes 1_{\mathcal{A}}}_{i} \otimes a \otimes \underbrace{1_{\mathcal{A}} \otimes \cdots \otimes 1_{\mathcal{A}}}_{k-i-1},
$$

for all $a \in \mathcal{L}$. From (6) and (9) we deduce:

$$
a\left(\varphi_{1} \star \cdots \star \varphi_{k}\right)=0_{\mathcal{A}}
$$

for all $a \in \mathcal{L}, k>1$ and $\varphi_{1}, \cdots, \varphi_{k} \in \mathcal{E}$ with the property $1_{\mathcal{A}} \varphi_{j}=0_{\mathcal{A}}, 1 \leq j \leq k$. Recall $E=\sum_{n \geq 0} 1_{\mathcal{S}_{n}}=1_{(\mathcal{P}, *)}$. Put $I:=\sum_{n \geq 1} 1_{\mathcal{S}_{n}}$, that means $E=\emptyset+I$, then $I^{\star n} a=0_{\mathcal{A}}$ for all $a \in \mathcal{L}$ and $n=0$ or $n>1$, by $(10) .{ }^{3}$ Define

$$
R:=\log E=\sum_{n \geq 1} \frac{(-1)^{n-1}}{n} I^{\star n},
$$

${ }^{3} I^{\star n}$ denotes the $n$-fold convolution product of $I$ with itself. 
then $R a=a$ for all $a \in \mathcal{L}$. Define the mapping

$$
\widehat{\downarrow}: \widehat{\mathcal{P}} \rightarrow \prod_{n=0}^{\infty}\left(\bigoplus_{k=0}^{n} K \mathcal{S}_{k} \otimes K \mathcal{S}_{n-k}\right)=\widehat{\mathcal{P} \otimes \mathcal{P}}
$$

as the continuous extension of $\downarrow$. For any sequence $\left(\alpha_{n}\right)_{n \geq 0}$ with $\alpha_{n} \in K \mathcal{S}_{n}$ the series $\alpha:=\sum_{n \geq 0} \alpha_{n}$ is convergent and

$$
\alpha \widehat{\downarrow}=\sum_{n \geq 0}\left(\alpha_{n} \downarrow\right) .
$$

From $1_{\mathcal{S}_{n} \downarrow}=\sum_{k=0}^{n} 1_{\mathcal{S}_{k}} \otimes 1_{\mathcal{S}_{n-k}}$ we deduce

$$
E \widehat{\downarrow}=E \otimes E=\sum_{n=0}^{\infty} \sum_{k=0}^{n} 1_{\mathcal{S}_{k}} \otimes 1_{\mathcal{S}_{n-k}} \in \widehat{\mathcal{P} \otimes \mathcal{P}} .
$$

It is well known that $R=\log E$ is primitive with respect to $\widehat{\downarrow}$, i.e.

$$
R \widehat{\downarrow}=R \otimes \emptyset+\emptyset \otimes R .
$$

Putting $\rho_{n}:=p_{n}(R)$, in particular $R=\sum_{n \geq 1} \rho_{n}$, we have now:

$$
\rho_{n} \downarrow=\rho_{n} \otimes \emptyset+\emptyset \otimes \rho_{n} .
$$

The elements $\rho_{n}$ are contained in the subalgebra of $(\mathcal{P}, \star)$ generated by all $1 \mathcal{S}_{n}$, i.e. in Solomons Algebra $\mathcal{D}=\bigoplus_{n \geq 0} \mathcal{D}_{n}$, which is contained in $\mathfrak{A}$ by 1.1. Again by 1.1 , we conclude for $n \geq 1$ and all $y \in \mathcal{A}_{n}$

$$
\begin{aligned}
(R y) \delta= & \left(\rho_{n} y\right) \delta=\left(\rho_{n} \downarrow\right)(y \delta)= \\
& =\rho_{n} y \otimes 1_{\mathcal{A}}+1_{\mathcal{A}} \otimes \rho_{n} y=R y \otimes 1_{\mathcal{A}}+1_{\mathcal{A}} \otimes R y,
\end{aligned}
$$

therefore $R \mathcal{A} \subseteq \mathcal{L}$ by the theorem of Friedrichs. Since $R a=a$ for all $a \in \mathcal{L}$ we get

Proposition 2.1 $R$ is a Lie projector, i.e. $R$ is an idempotent in $(\widehat{\mathcal{P}}, *)$ and $R \mathcal{A}\langle X\rangle=$ $\mathcal{L}\langle X\rangle$ for all sets $X$. Especially, $\rho_{n}$ is an idempotent in $\mathfrak{A}_{n}{ }^{4}$

We can easily deduce from 2.1:

$$
\mathcal{O}=R * \mathcal{P}=\bigoplus_{n \geq 1} \rho_{n} * K \mathcal{S}_{n} \quad \text { and } \quad \mathcal{O}_{n}=\rho_{n} * K \mathcal{S}_{n}
$$

In particular, $\mathcal{O}$ is a right ideal in $(\mathcal{P}, *)$. Concerning the proof, we remark that

$$
x * y=(x(y \mathrm{w})) \mathrm{st}=y \mathrm{w}(\mathrm{pol} x) \mathrm{st}
$$

\footnotetext{
${ }^{4}$ The elements $\rho_{n}$ first appeared in [12], by communication of a referee also in [2], [8]. Further, pol $R$ is the canonical projection $\pi_{1}$ from [10].
} 
for all $x \in \widehat{\mathcal{P}}$ and $y \in \mathcal{P}$, therefore

$$
R * y=(R(y \mathrm{w})) \mathrm{st} \in(\mathcal{P} \mathrm{w} \cap \mathcal{L}) \mathrm{st}=\mathcal{O},
$$

that means $R * \mathcal{P} \subseteq \mathcal{O}$. On the other hand, if $y \in \mathcal{O}$ then $y \mathrm{w} \in \mathcal{L}$ and $R * y=$ $(R(y \mathrm{w})) \mathrm{st}=y \mathrm{w} \mathrm{st}=y$.

\section{Multiplicative reciprocity}

We prove Schocker's theorem in a rather generalized version, which is more convenient for applications.

In analogy to $\delta^{(k)}$ we define recursively

$$
\downarrow^{(k)}:=\downarrow\left(\downarrow^{(k-1)} \otimes \mathrm{id}\right) .
$$

Then

$$
\downarrow^{(k)}: \mathcal{P} \rightarrow \mathcal{P}^{\otimes k}=\bigoplus_{n=0}^{\infty}\left(\bigoplus_{j_{1}+\cdots+j_{k}=n} K \mathcal{S}_{j_{1}} \otimes \cdots \otimes K \mathcal{S}_{j_{k}}\right)
$$

is a homogeneous algebra homomorphism. Further,

$$
\widehat{\downarrow}^{(k)}: \widehat{\mathcal{P}} \rightarrow \prod_{n=0}^{\infty}\left(\bigoplus_{j_{1}+\cdots+j_{k}=n} K \mathcal{S}_{j_{1}} \otimes \cdots \otimes K \mathcal{S}_{j_{k}}\right)=\widehat{\mathcal{P} \otimes k}
$$

denotes the continuous extension of $\downarrow^{(k)}$, that means $x{\downarrow^{(k)}}^{(k)} \sum_{n \geq 0} x_{n} \downarrow^{(k)}$ for $x=\sum_{n \geq 0} x_{n}, x_{n} \in K \mathcal{S}_{n}$. Similarly $\widehat{\mathrm{conv}}^{(k)}$ is the continuous extension of $\operatorname{conv}^{(k)}$ : $\mathcal{P}^{\otimes k} \rightarrow \mathcal{P}, z_{1} \otimes \cdots \otimes z_{k} \mapsto z_{1} \star \cdots \star z_{k}$. Put $\mathcal{O}_{n}:=\mathcal{O} \cap K \mathcal{S}_{n}$ and $\mathfrak{A}_{n}:=\mathfrak{A} \cap K \mathcal{S}_{n}$, then $\mathcal{O}=\bigoplus_{n \geq 1} \mathcal{O}_{n}$ and $\mathfrak{A}=\bigoplus_{n \geq 0} \mathfrak{A}_{n}$. We put $\widehat{\mathfrak{A}}:=\prod_{n \geq 0} \mathfrak{A}_{n}$ and $\widehat{\mathcal{O}}:=\prod_{n \geq 1} \mathcal{O}_{n}$. For the proof of Schocker's theorem we need a remarkable relationship between the convolution product on $\mathcal{P}$ and the coproduct $\downarrow$, the reciprocity law. We define a symmetric, non-degenerate bilinear form $(,)_{\mathcal{P}}$ on $\mathcal{P}$ by

$$
(\sigma, \tau)_{\mathcal{P}}:= \begin{cases}1 & \text { if } \sigma=\tau^{-1} \\ 0 & \text { otherwise }\end{cases}
$$

for all permutations $\sigma$ and $\tau$ and bilinear extension. A moment's reflection reveals

$$
(\alpha * \beta, \gamma)_{\mathcal{P}}=(\alpha, \beta * \gamma)_{\mathcal{P}}=(\beta, \gamma * \alpha)_{\mathcal{P}},
$$

for all $\alpha, \beta, \gamma \in \mathcal{P}$. There exists exactly one non-degenerate and symmetric bilinear form $(,)_{\mathcal{P} \otimes \mathcal{P}}$ on $\mathcal{P} \otimes \mathcal{P}$ with the property

$$
(\alpha \otimes \beta, \gamma \otimes \delta)_{\mathcal{P} \otimes \mathcal{P}}:=(\alpha, \gamma)_{\mathcal{P}}(\beta, \delta)_{\mathcal{P}},
$$

for all $\alpha, \beta, \gamma, \delta \in \mathcal{P}$. 
Reciprocity Law 3.1 (cf. [3]) For all $\alpha_{1}, \alpha_{2}, \beta \in \mathcal{P}$ :

$$
\left(\alpha_{1} \star \alpha_{2}, \beta\right)_{\mathcal{P}}=\left(\alpha_{1} \otimes \alpha_{2}, \beta \downarrow\right)_{\mathcal{P} \otimes \mathcal{P}}
$$

The following assertion is a little bit changed and more convenient formulation of Schocker's theorem 1.4.

Main Lemma 3.2 For all $x \in \widehat{\mathcal{P}}$ the following statements are equivalent:

(i) $x \in \widehat{\mathfrak{A}}$,

(ii) $\left(z_{1} \star \cdots \star z_{k}\right) * x=\left(\left(z_{1} \otimes \cdots \otimes z_{k}\right) *{ }_{\otimes} x \widehat{\downarrow}^{(k)}\right) \widehat{\operatorname{conv}}^{(k)}$

for all $z_{1}, \cdots, z_{k} \in \widehat{\mathcal{P}}$ and for all $k \in \mathbb{N}$,

(iii) $\left(z_{1} \star z_{2}\right) * x=\left(\left(z_{1} \otimes z_{2}\right) *{ }_{\otimes} x \widehat{\downarrow}\right)$ conv for all $z_{1}, z_{2} \in \widehat{\mathcal{P}}$,

(iv) $\left(z_{1} \star \cdots \star z_{k}\right) * x=\left(\left(z_{1} \otimes \cdots \otimes z_{k}\right) *_{\otimes} x \widehat{\downarrow}^{(k)}\right) \operatorname{conv}^{(k)}$

for all $z_{1}, \cdots, z_{k} \in \mathcal{P}$ and for all $k \in \mathbb{N}$,

(v) $\left(z_{1} \star z_{2}\right) * x=\left(\left(z_{1} \otimes z_{2}\right) *{ }_{\otimes} x \widehat{\downarrow}\right)$ conv for all $z_{1}, z_{2} \in \mathcal{P}$,

(vi) $(x * z) \widehat{\downarrow}=x \widehat{\downarrow} *{ }_{\otimes} z \widehat{\downarrow}$ for all $z \in \widehat{\mathcal{P}}$,

(vii) $(x * z) \widehat{\downarrow}=x \widehat{\downarrow} *{ }_{\otimes} z \downarrow$ for all $z \in \mathcal{P} .5$

Proof Let $x=\sum_{n \geq 0} x_{n} \in \widehat{\mathcal{P}}, x_{n} \in K \mathcal{S}_{n}$. Every statement (i) up to (vii) is true for $x$ if and only if it is true for all $x_{n}$. Therefore, we can assume $x \in K \mathcal{S}_{n}$ for some $n$. First we show the equivalence of (ii) up to (vii). Furthermore, we can assume $z_{1} \star \cdots \star z_{k}, z_{1} \star z_{2}, z \in K \mathcal{S}_{n}$ resp. In particular (ii) and (iv), (iii) and (v), (vi) and (vii) resp. are equivalent. (v) is a weakening of (iv). By induction on $k$ we show that (iv) follows from (v). For $k=1$ nothing is to prove. Let $k \geq 2$ and $z:=z_{1} \star \cdots \star z_{k-1}$. Using Sweedler's notation ([14]) we conclude:

$$
\begin{aligned}
\left(z_{1} \star \cdots\right. & \left.\star z_{k}\right) * x \\
& =\left(z \star z_{k}\right) * x \\
& =\left(\left(z \otimes z_{k}\right) *_{\otimes} x \downarrow\right) \mathrm{conv} \\
& =\sum\left(z * x^{(1)} \otimes z_{k} * x^{(2)}\right) \mathrm{conv} \\
& =\sum\left(\left(\left(z_{1} \otimes \cdots \otimes z_{k-1}\right) *_{\otimes} x^{(1)} \downarrow^{(k-1)}\right) \operatorname{conv}^{(k-1)} \otimes z_{k} * x^{(2)}\right) \mathrm{conv} \\
& =\sum\left(\left(\left(z_{1} \otimes \cdots \otimes z_{k-1}\right) *_{\otimes} x^{(1)} \downarrow^{(k-1)}\right) \otimes z_{k} * x^{(2)}\right) \mathrm{conv}^{(k)} \\
& =\left(\left(\left(z_{1} \otimes \cdots \otimes z_{k-1}\right) \otimes z_{k}\right) *{ }_{\otimes} x \downarrow\left(\downarrow{ }^{(k-1)} \otimes \mathrm{id}\right)\right) \operatorname{conv}^{(k)} \\
& =\left(\left(z_{1} \otimes \cdots \otimes z_{k}\right) *{ }_{\otimes} x \downarrow^{(k)}\right) \operatorname{conv}^{(k)}
\end{aligned}
$$

${ }^{5}$ Cf. [9], where (vii) is shown for all $x, z \in \mathfrak{A}$ (Theorem 10). 
Altogether, (ii) up to (v) are equivalent. To prove equivalence of (v) and (vii), we argue as follows: on the one hand we have for all $z_{1}, z_{2} \in \mathcal{P}$

$$
\begin{aligned}
\left((x * z) \downarrow, z_{1} \otimes z_{2}\right)_{\mathcal{P} \otimes \mathcal{P}} & =\left(x * z, z_{1} \star z_{2}\right)_{\mathcal{P}} \\
& =\left(z,\left(z_{1} \star z_{2}\right) * x\right)_{\mathcal{P}}
\end{aligned}
$$

and on the other hand

$$
\begin{aligned}
\left(x \downarrow * \otimes z \downarrow, z_{1} \otimes z_{2}\right)_{\mathcal{P} \otimes \mathcal{P}} & =\left(z \downarrow,\left(z_{1} \otimes z_{2}\right) *_{\otimes} x \downarrow\right)_{\mathcal{P} \otimes \mathcal{P}} \\
& =\left(z,\left(\left(z_{1} \otimes z_{2}\right) *_{\otimes} x \downarrow\right) \text { conv }\right)_{\mathcal{P}} .
\end{aligned}
$$

Therefore (vii) is true, if and only if for all $z_{1}, z_{2} \in \mathcal{P}$,

$$
\left(z_{1} \star z_{2}\right) * x=\left(\left(z_{1} \otimes z_{2}\right) *_{\otimes} x \downarrow\right) \text { conv }
$$

i.e if and only if (v) is true. Now the equivalence of (ii) up to (vii) is shown.

Assume (ii), in particular for all $k \geq 0$ :

$$
(\overbrace{R \star \cdots \star R}^{k}) * x=\left((\overbrace{R \otimes \cdots \otimes R}^{k}) * \otimes \downarrow^{(k)}\right) \widehat{\operatorname{conv}}^{(k)} .
$$

Since $R * \mathcal{P}=\mathcal{O}$, we have $R^{\star k} * x \in \mathcal{O}^{\star k}$. We conclude:

$$
\begin{aligned}
x & =1_{(\widehat{\mathcal{P}}, *)} * x \\
& =\exp R * x \\
& =\sum_{k \geq 0} \frac{1}{k !} R^{\star k} * x \\
& =\sum_{k=0}^{n} \frac{1}{k !} R^{\star k} * x \in \sum_{k=0}^{n} \mathcal{O}^{\star k} \subseteq \mathfrak{A} \subseteq \widehat{\mathfrak{A}} .
\end{aligned}
$$

Remains to show that (v) is a consequence of (i). We prove this under the additional assumption that $x$ is contained in the convolution subalgebra of $\mathfrak{A}$, generated by the primitive elements of $\mathcal{O}^{6}$. By linearity we may assume that $x=y_{1} \star \cdots \star y_{l}$ is a convolution product of primitive elements of $\mathcal{O}$. Let $X$ be an infinite set and $\mathcal{A}:=$ $\mathcal{A}\langle X\rangle$. Call $Y_{j}:=\operatorname{pol} y_{j}, Z_{i}:=\operatorname{pol} z_{i}$ and $\mathfrak{Y}:=($ pol $\otimes$ pol $)(x \downarrow)$, the endomorphisms, induced by Polya action from $z_{i}, y_{j}, x \downarrow$ on $\mathcal{A}, \mathcal{A} \otimes \mathcal{A}$ resp. Now (v) is equivalent to:

$$
\left(Y_{1} \star \cdots \star Y_{l}\right)\left(Z_{1} \star Z_{2}\right)=\left(\mathfrak{Y}\left(Z_{1} \otimes Z_{2}\right)\right) \text { conv. }
$$

On the other hand,

$$
\left(Y_{1} \star \cdots \star Y_{l}\right) \delta=\delta \mathfrak{Y},
$$

${ }^{6}$ By [9] $\mathcal{O}=\operatorname{Prim} \mathfrak{A}$. 
by 1.1 . As an easy consequence we get

$$
\begin{aligned}
\left(Y_{1} \star \cdots\right. & \left.\star Y_{l}\right)\left(Z_{1} \star Z_{2}\right) \\
& =\left(Y_{1} \star \cdots \star Y_{l}\right) \delta\left(Z_{1} \otimes Z_{2}\right) \text { conc } \\
& =\delta \mathfrak{Y}\left(Z_{1} \otimes Z_{2}\right) \text { conc } \\
& =\left(\mathfrak{Y}\left(Z_{1} \otimes Z_{2}\right)\right) \text { conv. }
\end{aligned}
$$

It remains to show that all elements of $\mathcal{O}$ are primitive. Let $x$ be an element of $\mathcal{O}$. Since $R \in \widehat{\mathcal{O}}$ and $x=R * x$ on account of (12), we can apply (vi), and conclude due to (11):

$$
x \downarrow=(R * x) \downarrow=R \widehat{\downarrow} *{ }_{\otimes} x \downarrow=(R * x) \otimes \emptyset+\emptyset \otimes(R * x)=x \otimes \emptyset+\emptyset \otimes x .
$$

\section{Multiplication rules}

Corollary 4.1 If $y$ is a primitive element in $(\mathcal{P}, \downarrow)$ and $x \in \widehat{\mathfrak{A}}$, then $x * y$ is also primitive. In particular, Prim $\mathcal{P}$ is an $\widehat{\mathfrak{A}}$-left module.

Proof In Sweedler's notation we have $z \downarrow=z \otimes \emptyset+\emptyset \otimes z+\sum z^{(1)} \otimes z^{(2)}$ for all $z \in \mathcal{P}$, where $z^{(1)}, z^{(2)} \neq \emptyset$. The statement now follows from 3.2, (vii).

Corollary 4.2 If $z_{1}, z_{2}$ are elements of $\widehat{\mathcal{P}}$ with the property $p_{0}\left(z_{1}\right)=0=p_{0}\left(z_{2}\right)$, and if $x \in \widehat{\mathfrak{A}}$ is primitive, then $\left(z_{1} \star z_{2}\right) * x=0_{\widehat{\mathcal{P}}}$. Further, $\widehat{\mathcal{O}}$ is the set of all primitive elements of $\widehat{\mathfrak{A}}$. In particular, $\mathcal{O}$ is the set of primitive elements of $\mathfrak{A}$ (cf. [9]).

Proof From 3.2, (iii) we conclude

$$
\left(z_{1} \star z_{2}\right) * x=\left(z_{1} * x\right) \star\left(z_{2} * \emptyset\right)+\left(z_{1} * \emptyset\right) \star\left(z_{2} * x\right)=0_{\widehat{\mathcal{P}}}
$$

The second statement follows from [9]. For convenience, we give the short argument. The end of the proof of 3.2 shows in particular:

$$
\widehat{\mathcal{O}} \subseteq \operatorname{Prim}(\widehat{\mathfrak{A}}, \widehat{\downarrow})
$$

An element $x \in \widehat{\mathcal{P}}$ is primitive if and only if $p_{n}(x)$ is primitive for all $n$. In particular, $p_{0}(x)=0$. Now let $x \in \operatorname{Prim} \widehat{\mathfrak{A}}$. Then

$$
x=E * x=\exp R * x=\sum_{n \geq 0} \frac{1}{n !} R^{\star n} * x=R * x,
$$

finally $\widehat{\mathcal{O}}=R * \widehat{\mathfrak{A}} \supseteq R * \operatorname{Prim} \widehat{\mathfrak{A}}=\operatorname{Prim} \widehat{\mathfrak{A}}$.

A useful consequence of 3.2, (ii) is the following multiplication rule for all $\alpha_{1}, \cdots$, $\alpha_{l} \in \widehat{\mathcal{P}}$ and $\beta_{1}, \cdots, \beta_{k} \in \widehat{\mathcal{O}}$ :

$$
\left(\alpha_{1} \star \cdots \star \alpha_{l}\right) *\left(\beta_{1} \star \cdots \star \beta_{k}\right)=\sum_{J_{1}, \cdots, J_{l}}\left(\alpha_{1} * \beta_{J_{1}}\right) \star \cdots \star\left(\alpha_{l} * \beta_{J_{l}}\right) .
$$


The summation is extended over all pairwise disjoint subsets $J_{1}, \cdots, J_{l}$ of $[k]$ such that $J_{1} \cup \cdots \cup J_{l}=[k]$; if $J=\left\{j_{1}, \cdots, j_{m}\right\} \subseteq[k]$ with $j_{1}<\cdots<j_{m}$, then $\beta_{J}=$ $\beta_{j_{1}} \star \cdots \star \beta_{j_{m}}$, and if $J=\emptyset$, then $\beta_{J}=1_{(\mathcal{P}, \star)}=\emptyset \in \mathcal{S}_{0}$. Patras and Reutenauer ([9], Theorem 10) have shown that $\mathfrak{A}$ is a subalgebra of $(\mathcal{P}, *)$. This is also a consequence of (15): if $\alpha_{1}, \cdots, \beta_{k} \in \mathcal{O}$ the product in (15) is an element of $\mathfrak{A}$, since $\mathcal{O}=R * \mathcal{P}$ is a right ideal of $(\mathcal{P}, *)$. Two special cases are of interest:

$$
\left(\alpha_{1} \star \cdots \star \alpha_{k}\right) *\left(\beta_{1} \star \cdots \star \beta_{k}\right)=\sum_{\sigma \in \mathcal{S}_{k}}\left(\alpha_{1} * \beta_{1 \sigma}\right) \star \cdots \star\left(\alpha_{l} * \beta_{l \sigma}\right) .
$$

If $p_{0}\left(\alpha_{1}\right)=\cdots=p_{0}\left(\alpha_{l}\right)=0_{\widehat{\mathcal{P}}}$ and $l>k$ :

$$
\left(\alpha_{1} \star \cdots \star \alpha_{l}\right) *\left(\beta_{1} \star \cdots \star \beta_{k}\right)=0_{\widehat{\mathcal{P}}}
$$

Denote some simple consequences of (15), (16) and (17):

$-R^{\star n} * R^{\star k}=0_{\widehat{\mathcal{P}}}=I^{\star n} * R^{\star k} \quad$ if $n>k$,

$-R^{\star n} * R^{\star n}=n ! R^{\star n}$,

$-I^{\star l} * R^{\star k}=l ! \mathfrak{S}_{k}^{(l)} R^{\star k} \quad$ if $l \leq k$,

$-R * R^{\star k}=\left(\sum_{l=1}^{k}(-1)^{l-1}(l-1) ! \mathfrak{S}_{k}^{(l)}\right) R^{\star k}=0_{\widehat{\mathcal{P}}} \quad$ if $1<k$,

$-R^{\star n} * R^{\star k}=0_{\widehat{\mathcal{P}}} \quad$ if $n \neq k$.

Here $\mathfrak{S}_{k}^{(l)}$ is a Stirling number of the second kind, i.e. the number of ways of partitioning of $[k]$ into $l$ non-empty subsets. The fourth equation follows easily from $\mathfrak{S}_{k}^{(l)}=l \mathfrak{S}_{k-1}^{(l)}+\mathfrak{S}_{k-1}^{(l-1)}$ for $k>l$. As a consequence, we get:

Proposition 4.3 The elements $1 / k ! R^{\star k}$ of $\widehat{\mathcal{O}}$ constitute a system of pairwise orthogonal idempotents of $(\widehat{\mathcal{P}}, *)$, summing up to the neutral element $E=\exp R$ of $(\widehat{\mathcal{P}}, *)$ ([10]). In particular, putting $\mathcal{O}^{(k)}:=R^{\star k} * \mathfrak{A}$,

$$
\mathfrak{A}=\bigoplus_{k \geq 0} \mathcal{O}^{(k)}
$$

is a direct decomposition of $(\mathfrak{A}, *)$ into right ideals. Moreover, $\sum_{k=1}^{n} \mathcal{O}^{(k)}$ is an ideal of $(\mathfrak{A}, *)$ for all $n \in \mathbb{N}$ and

$$
\mathcal{O}^{(k)}=\left\langle\sum_{\sigma \in \mathcal{S}_{k}} \alpha_{1 \sigma} \star \cdots \star \alpha_{k \sigma} \mid \alpha_{1}, \cdots, \alpha_{k} \in \mathcal{O}\right\rangle_{K}
$$

Proof Put $\mathcal{O}^{\star n}:=\overbrace{\mathcal{O} \star \cdots \star \mathcal{O}}^{n}$, then by $(17)$

$$
\mathcal{O}^{\star n}=E * \mathcal{O}^{\star n}=\sum_{k \geq 0} \frac{1}{k !} R^{\star k} * \mathcal{O}^{\star n}=\sum_{k=0}^{n} R^{\star k} * \mathcal{O}^{\star n} \subseteq \sum_{k=0}^{n} \mathcal{O}^{(k)}
$$


therefore $\sum_{k=0}^{n} \mathcal{O}^{\star k}=\sum_{k=0}^{n} \mathcal{O}^{(k)}$. Applying $R^{\star n}$ on this equation by Polya action we conclude $R^{\star n} * \mathcal{O}^{\star n}=\mathcal{O}^{(n)}$. The assertion (18) now follows from (16). By (17),

$$
\mathcal{O}^{\star l} * \mathcal{O}^{\star k}=\left\{0_{\widehat{\mathcal{P}}}\right\} \quad \text { if } l>k .
$$

In particular, $\sum_{k=1}^{n} \mathcal{O}^{(k)}$ is an ideal in $(\mathfrak{A}, *)$.

\section{The endomorphism $\Theta$ of $\mathfrak{A}$}

The next statement describes another combinatorial property of the Patras-Reutenauer algebra.

Theorem 5.1 Let $x \in \mathfrak{A}_{n}$, then $(x \mathrm{w})_{J}$ st is an element of $\mathfrak{A}_{|J|}$ and depends only on $|J|$, for all $J \subseteq[n]$.

Proof As a convolution algebra, $\mathfrak{A}$ is generated by it's primitive elements ([9] or 4.2). If $\alpha_{1}, \cdots, \alpha_{k} \in \operatorname{Prim} \mathfrak{A}$ then

$$
\left(\alpha_{1} \star \cdots \star \alpha_{k}\right) \downarrow=\sum_{J \subseteq[k]} \alpha^{J} \otimes \alpha^{[k] \backslash J},
$$

where $\alpha^{J}=\alpha_{j_{1}} \star \cdots \star \alpha_{j_{l}}$ for $J=\left\{j_{1}, \cdots, j_{l}\right\}, j_{1}<\cdots<j_{l}$. Therefore $\mathfrak{A} \downarrow \subseteq \mathfrak{A} \otimes \mathfrak{A}$ and $\mathfrak{A}_{n} \downarrow \subseteq \bigoplus \mathfrak{A}_{j} \otimes \mathfrak{A}_{n-j}$. Let $x=\sum_{\sigma \in \mathcal{S}_{n}} k_{\sigma} \sigma$, then by (3)

$$
x \downarrow=\sum_{j=0}^{n} \sum_{\sigma \in \mathcal{S}_{n}} k_{\sigma}\left((\sigma \mathrm{w})_{[j]} \mathrm{st} \otimes(\sigma \mathrm{w})_{[n] \backslash[j]} \mathrm{st}\right) .
$$

For all $J \subseteq[n]$ with $|J|=n-1$ now follows by 1.3 :

$$
\begin{aligned}
(x \mathrm{w})_{J} \mathrm{st} \otimes 1_{\mathcal{S}_{1}} & =\left(\sum_{\sigma \in \mathcal{S}_{n}} k_{\sigma}(\sigma \mathrm{w})_{J} \mathrm{st}\right) \otimes 1_{\mathcal{S}_{1}} \\
& =\sum_{\sigma \in \mathcal{S}_{n}} k_{\sigma}\left((\sigma \mathrm{w})_{J} \mathrm{st} \otimes(\sigma \mathrm{w})_{[n] \backslash J \mathrm{st})}\right. \\
& =\sum_{\sigma \in \mathcal{S}_{n}} k_{\sigma}\left((\sigma \mathrm{W})_{[n-1]} \mathrm{st} \otimes(\sigma \mathrm{W})_{[n] \backslash[n-1]} \mathrm{st}\right) \\
& =\left(\sum_{\sigma \in \mathcal{S}_{n}} k_{\sigma}(\sigma \mathrm{W})_{[n-1]} \mathrm{st}\right) \otimes 1_{\mathcal{S}_{1}} \\
& =(x \mathrm{w})_{[n-1]} \mathrm{st} \otimes 1_{\mathcal{S}_{1}} .
\end{aligned}
$$

Since the summand for $j=n-1$ in (19) is an element of $\mathfrak{A}_{n-1} \otimes \mathfrak{A}_{1}=\mathfrak{A}_{n-1} \otimes 1_{\mathcal{S}_{1}}$, we conclude

$$
(x \mathrm{w})_{J} \mathrm{st} \otimes 1_{\mathcal{S}_{1}}=a \otimes 1_{\mathcal{S}_{1}}=(x \mathrm{w})_{[n-1]} \mathrm{st} \otimes 1_{\mathcal{S}_{1}},
$$


for some suitable $a \in \mathfrak{A}_{n-1}$. A simple argument, using the basis $\mathcal{S}$ of $\mathcal{P}$, shows

$$
(x \mathrm{~W})_{J} \mathrm{st}=a=(x \mathrm{~W})_{[n-1]} \mathrm{st} .
$$

We have proved the statement for $|J|=n-1$. Let $L, L^{\prime}$ be different subsets of $[n]$ such that $|L|=\left|L^{\prime}\right| \leq n-2$. Take $i \in L \backslash L^{\prime}$ and $j \in L^{\prime} \backslash L$, put $L^{\prime \prime}:=(L \backslash\{i\}) \cup$ $\{j\}$, then $i \notin L^{\prime} \cup L^{\prime \prime}$ and $\left|L \cup L^{\prime \prime}\right|=|L \cup\{j\}|=|L|+1 \leq n-1$. Therefore exist $J_{1}, J_{2} \subseteq[n]$ with the property $\left|J_{1}\right|=n-1=\left|J_{2}\right|$ and $L^{\prime} \cup L^{\prime \prime} \subseteq J_{1}, L \cup L^{\prime \prime} \subseteq J_{2}$. If $M \subseteq N \subseteq[n]$, then for all $\sigma \in \mathcal{S}_{n}$ we have the transitivity rule:

$$
\left.(\sigma \mathrm{w})_{M} \mathrm{st}=\left((\sigma \mathrm{w})_{N}\right)_{M}\right) \mathrm{st}=\left((\sigma \mathrm{w})_{N} \varphi\right)_{M \varphi} \mathrm{st}=\left(\left((\sigma \mathrm{w})_{N} \mathrm{st}\right) \mathrm{w}\right)_{M \varphi} \mathrm{st},
$$

where $\varphi$ is an algebra endomorphism of $\mathcal{A}\langle\mathbb{N}\rangle$, such that $\varphi$ induces the uniquely determined order isomorphism of $N$ onto $\{1, \cdots,|N|\}$. For example,

$$
(x \mathrm{w})_{L} \mathrm{st}=\left(\left((x \mathrm{w})_{J_{1}} \mathrm{st}\right) \mathrm{w}\right)_{L \varphi} \mathrm{st} .
$$

Because $(x \mathrm{w})_{J_{1}}$ st $=(x \mathrm{w})_{J_{2}}$ st $\in \mathfrak{A}_{n-1}$, we conclude by induction

$$
(x \mathrm{w})_{L} \mathrm{st}=(x \mathrm{w})_{L^{\prime \prime}} \mathrm{st}=(x \mathrm{w})_{L^{\prime}} \mathrm{st} \in \mathfrak{A}_{|L|} .
$$

We define a linear mapping $\Theta: \mathcal{P} \rightarrow \mathcal{P}, \sigma \mapsto \sigma \Theta:=(\sigma \mathrm{w})_{[n-1]}$ st, for all $\sigma \in \mathcal{S}_{n}$. Roughly spoken, $\sigma \Theta$ emerges from $\sigma \in \mathcal{S}_{n}$ by striking out the letter $n$ in the image line of $\sigma$, for example, $13247865 \Theta=1324765$. Then $\mathfrak{A} \Theta \subseteq \mathfrak{A}$ by 5.1. As a matter of fact, $(x \mathrm{~W})_{J}$ st $=x \Theta^{n-|J|}$, for all $x \in \mathfrak{A}_{n}$ and all $J \subseteq[n]$.

Proposition $5.2 \Theta: \mathfrak{A} \rightarrow \mathfrak{A}$ is a homomorphism for the inner product. In particular, $((x * y) \mathrm{w})_{J} \mathrm{st}=(x \mathrm{w})_{J} \mathrm{st} *(y \mathrm{w})_{J} \mathrm{st}$, for all $x, y \in \mathfrak{A}_{n}$ and all $J \subseteq[n]$.

Proof Let $x=\sum_{\sigma \in \mathcal{S}_{n}} k_{\sigma} \sigma$ and $y=\sum_{\sigma \in \mathcal{S}_{n}} l_{\sigma} \sigma$ be elements of $\mathfrak{A}_{n}$. Put $x * y=$ $\sum_{\sigma \in \mathcal{S}_{n}} m_{\sigma} \sigma$. Recall that $\mathfrak{A}$ is a subalgebra of $(\mathcal{P}, *)$. By Schocker's theorem (or [9], Theorem 10) $(x * y) \downarrow=x \downarrow *_{\otimes} y \downarrow$, and we conclude by 1.3

$$
\begin{aligned}
((x & * y) \mathrm{w})_{[n-1]} \mathrm{st} \otimes 1_{\mathcal{S}_{1}} \\
\quad & =\sum_{\sigma \in \mathcal{S}_{n}} m_{\sigma}\left((\sigma \mathrm{w})_{[n-1]} \mathrm{st} \otimes(\sigma \mathrm{w})_{[n] \backslash[n-1]} \mathrm{st}\right) \\
& =\sum_{\rho, \tau \in \mathcal{S}_{n}} k_{\rho} l_{\tau}\left((\rho \mathrm{w})_{[n-1]} \mathrm{st} *(\tau \mathrm{w})_{[n-1]} \mathrm{st} \otimes 1_{\mathcal{S}_{1}}\right) \\
& =\left(\left(\sum_{\rho \in \mathcal{S}_{n}} k_{\rho}(\rho \mathrm{w})_{[n-1]} \mathrm{st}\right) *\left(\sum_{\tau \in \mathcal{S}_{n}} k_{\tau}(\tau \mathrm{w})_{[n-1]} \mathrm{st}\right)\right) \otimes 1_{\mathcal{S}_{1}} \\
& =\left((x \mathrm{w})_{[n-1]} \mathrm{st} *(y \mathrm{w})_{[n-1]} \mathrm{st}\right) \otimes 1_{\mathcal{S}_{1}},
\end{aligned}
$$

therefore $(x * y) \Theta=x \Theta * y \Theta$. 
Proposition 5.3 The mapping $\Theta$ induces a derivation of the convolution algebra $(\mathfrak{A}, \star)$.

Proof Let $n \in \mathbb{N}, x \in \mathfrak{A}_{j}$ and $y \in \mathfrak{A}_{n-j}$. Put $v:=1.2 . \cdots . n \in \mathbb{N}^{*}$ and $u:=$ 1.2. ... . $(n-1) \in \mathbb{N}^{*}$. Then

$$
(x \star y) \mathrm{w}=(x \star y) 1.2 . \cdots . n=\sum_{\substack{J \subseteq[n] \\|J|=j}}\left(x v_{J}\right)\left(y v_{[n] \backslash J}\right),
$$

for example by (7). Observe that $(x \star y) \Theta=x \star y \Theta+x \Theta \star y$ is equivalent to $(x \star y) \mathrm{w}_{[n-1]}=(x \star y) \Theta \mathrm{w}=(x \star y \Theta) \mathrm{w}+(x \Theta \star y) \mathrm{w}$. On the one hand, we have

$$
\begin{aligned}
(x & \star y) \mathrm{w}_{[n-1]} \\
& =\sum_{\substack{J \subseteq \subseteq[n] \\
|J|=j}}\left(\left(x v_{J}\right)\left(y v_{[n] \backslash J}\right)\right)_{[n-1]} \\
& =\sum_{\substack{n \notin J \subseteq[n] \\
|J|=j}}\left(x v_{J}\right)\left(y v_{[n] \backslash J}\right)_{[n-1]}+\sum_{\substack{n \in J \subseteq[n] \\
|J|=j}}\left(x v_{J}\right)_{[n-1]}\left(y v_{[n] \backslash J}\right) .
\end{aligned}
$$

For $n \in J \subseteq[n]$ with $|J|=j$ call $\Phi$ any algebra endomorphism of $\mathcal{A}\langle\mathbb{N}\rangle$, such that $\Phi$ induces the uniquely determined order preserving bijection of $J$ onto [ $j]$. Then,

$$
x \Theta u_{J \backslash\{n\}} \Phi=x \Theta \mathrm{w}=(x \mathrm{w})_{[j-1]}=\left(x v_{J}\right)_{[n-1]} \Phi,
$$

therefore $x \Theta u_{J \backslash\{n\}}=\left(x v_{J}\right)_{[n-1]}$, analogously $y \Theta u_{[n] \backslash J}=\left(y v_{[n] \backslash J}\right)_{[n-1]}$ if $n \notin J$. To finish the proof, we conclude on the other hand

$$
\begin{aligned}
(x & \star y \Theta) \mathrm{w}+(x \Theta \star y) \mathrm{w} \\
& =\sum_{\substack{L \subseteq[n-1] \\
|L|=j}}\left(x u_{L}\right)\left(y \Theta u_{[n-1] \backslash L}\right)+\sum_{\substack{M \subseteq[n-1] \\
|M|=j-1}}\left(x \Theta u_{M}\right)\left(y u_{[n-1] \backslash M}\right) \\
& =\sum_{\substack{n \notin J \subseteq[n] \\
|J|=j}}\left(x u_{J}\right)\left(y \Theta u_{[n] \backslash J}\right)+\sum_{\substack{n \in J \subseteq[n] \\
|J|=j}}\left(x \Theta u_{J \backslash\{n\}}\right)\left(y v_{[n] \backslash J}\right) \\
& =\sum_{\substack{n \notin J \subseteq[n] \\
|J|=j}}\left(x u_{J}\right)\left(\left(y v_{[n] \backslash J}\right)_{[n-1]}\right)+\sum_{\substack{n \in J \subseteq[n] \\
|J|=j}}\left(\left(x v_{J}\right)_{[n-1]}\right)\left(y v_{[n] \backslash J}\right),
\end{aligned}
$$

and that was to be shown.

Next we prove that $\Theta$ induces an epimorphism of $\mathfrak{A}_{n}$ onto $\mathfrak{A}_{n-1}$. In particular, $\left(\mathfrak{A}_{n-1}, *\right)$ is isomorphic to a factor algebra of $\left(\mathfrak{A}_{n}, *\right)$. We first describe a system of generators for the vector space $\mathfrak{A}$. Recall that $\mathcal{O}$ is a homogeneous subspace of $\mathfrak{A}$, 
i.e.

$$
\mathcal{O}=\bigoplus_{k \geq 1} \mathcal{O}_{n},
$$

where $\mathcal{O}_{n}:=\mathcal{O} \cap K \mathcal{S}_{n}$. The elements of $\mathcal{O}_{n}$ are called homogeneous of degree $n$. Obviously, the set of all products $\alpha_{1} \star \cdots \star \alpha_{n}$, where $\alpha_{1}, \cdots, \alpha_{n}$ are homogeneous elements of $\mathcal{O}$, is a system of linear generators of $\mathfrak{A}$. Call $\mathcal{G}$ the set of all products

$$
\alpha_{1,1} \star \cdots \star \alpha_{1, j_{1}} \star \alpha_{2,1} \star \cdots \star \alpha_{2, j_{2}} \star \cdots \star \alpha_{l, 1} \star \cdots \star \alpha_{l, j_{l}},
$$

where $l \in \mathbb{N}$ and $\alpha_{i, k} \in \mathcal{O}_{i}$. Let $\mathcal{G}^{n}, \mathcal{G}_{n}$ resp. be the set of all such elements with the property $j_{1}+j_{2}+\cdots+j_{l}=n, 1 \cdot j_{1}+2 \cdot j_{2}+\cdots+l \cdot j_{l}=n$ resp., then $\mathcal{G}_{n} \subseteq \mathfrak{A}_{n}$. Finally put $\mathcal{O}^{<n>}$ the subspace generated by $\mathcal{G}^{n}$. By (16), (17) and 4.3 we get:

$$
R^{\star n} * \mathcal{O}^{<m>}= \begin{cases}\mathcal{O}^{(n)} & \text { if } n=m, \\ \left\{0_{\mathcal{P}}\right\} & \text { if } n>m .\end{cases}
$$

We conclude

$$
\sum_{k=0}^{n} \mathcal{O}^{<k>}=E \sum_{k=0}^{n} \mathcal{O}^{<k>}=\sum_{j=0}^{n}(j !)^{-1} R^{\star j} \sum_{k=0}^{n} \mathcal{O}^{<k>} \supseteq \sum_{k=0}^{n} \mathcal{O}^{(k)} .
$$

In particular, $\mathfrak{A}=\sum_{k \geq 0} \mathcal{O}^{<k>}$ by 4.3 , i.e. $\mathcal{G}$ generates the vector space $\mathfrak{A}$, and $\mathcal{G}_{n}$ is a generating system for $\mathfrak{A}_{n}$, since $\mathfrak{A}=\bigoplus \mathfrak{A}_{n}$. Recall the Specht-Wever element

$$
\omega_{n}=\sum_{\pi \in \mathcal{V}_{n}}(-1)^{1 \pi^{-1}-1} \pi,
$$

where $\mathcal{V}_{n}$ is the set of all valley permutations in $\mathcal{S}_{n}$ (cf. [3]). A permutation $\pi$ is in $\mathcal{V}_{n}$ if

$$
1 \pi>2 \pi>\cdots>k \pi>(k+1) \pi=1<(k+2) \pi<\cdots<n \pi .
$$

Polya action of $\omega_{n}$ creates left normed Lie monomials:

$$
\omega_{n} x_{1} \cdots x_{n}=\left[\cdots\left[\left[x_{1}, x_{2}\right], x_{3}\right] \cdots, x_{n}\right],
$$

for all words $x_{1} \cdots x_{n}$ of length $n$ over an alphabet $X$, e.g. by [6]. By the DynkinSpecht-Wever theorem we have $\omega_{n} * \omega_{n}=n \cdot \omega_{n}$. Since the left normed Lie monomials generate the Lie algebra $\mathcal{L}\langle X\rangle$, we conclude from $\omega_{n} * \sigma=\left(\omega_{n}(\sigma \mathrm{w})\right)$ st:

$$
\mathcal{O}_{n}=\omega_{n} * K \mathcal{S}_{n}=\omega_{n} * \mathfrak{A}_{n} .
$$

In particular, $\mathcal{O}_{1}=\omega_{1} * K \mathcal{S}_{1}=\left\langle\omega_{1}\right\rangle_{K}$. Therefore we may assume

$$
\alpha_{1,1}=\cdots=\alpha_{1, j_{1}}=\omega_{1},
$$

for all elements (21) of $\mathcal{G}_{n}$. From the definition of $\omega_{n}$ easily follows $\omega_{1} \Theta=\emptyset \in \mathcal{S}_{0}$ and

$$
\omega_{n} \Theta=0_{\mathcal{P}} \quad \text { for } n \geq 2,
$$


in particular $\mathcal{O}_{n}=\omega_{n} * \mathfrak{A}_{n} \subseteq \operatorname{ker} \Theta$ for $n \geq 0$ by 5.2, where $\operatorname{ker} \Theta$ denotes the kernel of $\Theta$. Together with 5.3 this leads to

$$
\begin{aligned}
\omega_{1}^{\star j_{1}} \star & \alpha_{2,1} \star \cdots \star \alpha_{2, j_{2}} \star \cdots \star \alpha_{l, 1} \star \cdots \star \alpha_{l, j_{l}} \Theta \\
& =j_{1} \omega_{1}^{\star\left(j_{1}-1\right)} \star \alpha_{2,1} \star \cdots \star \alpha_{2, j_{2}} \star \cdots \star \alpha_{l, 1} \star \cdots \star \alpha_{l, j_{l}} .
\end{aligned}
$$

Therefore $\mathcal{G} \Theta$ generates $\mathfrak{A}$ and $\mathcal{G}_{n} \Theta$ generates $\mathfrak{A}_{n-1}$. For all $a \in \mathfrak{A}_{n-1}$ and all $b=$ $\sum_{\sigma \in \mathcal{S}_{n}} c_{\sigma} \sigma \in \mathfrak{A}_{n}$,

$$
\begin{aligned}
\left(\omega_{1} \star a\right) * b & =\left(\left(\omega_{1} \otimes a\right) *{ }_{\otimes} b \downarrow\right) \text { conv } \\
& =\left(\omega_{1} *\left(\sum_{\sigma \in \mathcal{S}_{n}} c_{\sigma}\right) 1_{\mathcal{S}_{1}}\right) \star(a * b \Theta) \in \omega_{1} \star \mathfrak{A}_{n-1},
\end{aligned}
$$

by 3.2. Observing that $\Theta: \omega_{1} \star \mathfrak{A}_{n-1} \rightarrow \mathfrak{A}_{n-1}$ is a bijection, we have shown:

Proposition $5.4 \Theta: \mathfrak{A} \rightarrow \mathfrak{A}$ is surjective. In particular, $\mathfrak{A}_{n} \Theta=\mathfrak{A}_{n-1}$. Furthermore, $\omega_{1} \star \mathfrak{A}_{n-1}$ is a right ideal in $\left(\mathfrak{A}_{n}, *\right)$ and

$$
\mathfrak{A}_{n}=\left(\omega_{1} \star \mathfrak{A}_{n-1}\right) \oplus\left(\mathfrak{A}_{n} \cap \operatorname{ker} \Theta\right)
$$

\section{Concluding remarks}

By work of Loïc Foissy Prim $\mathcal{P}$ is a free Lie algebra with respect to convolution [4]. $\mathcal{O}$ is a Lie subalgebra of $\operatorname{Prim} \mathcal{P}$, since $(\mathfrak{A}, \star, \downarrow)$ is a Bialgebra and $\mathcal{O}=$ Prim $\mathfrak{A}$ ([9]). Therefore $\mathcal{O}$ is a free Lie algebra by a theorem of Shirshov/Witt (cf. [11]). As a consequence, $\mathfrak{A}$ is a free associative algebra, since $\mathfrak{A}$ is a universal envelopping algebra of $\mathcal{O}$ ([9]). The direct decomposition of $\mathfrak{A}$ in 4.3 then follows from the Poincaré-Birkhoff-Witt theorem. Schocker's result leads to the ideal properties of the subspaces $\mathcal{O}^{(l)}$. The homomorphism $\Theta$, described in 5.2, induces a homomorphism between Solomon's algebras $\mathcal{D}_{n}$ and $\mathcal{D}_{n-1}$, which was first studied in [1].

\section{References}

1. Bergeron, F., Garsia, A.M., Reutenauer, C.: Homomorphisms between Solomon's descent algebras. J. Algebra 150, 503-519 (1992)

2. Bialynicki-Birula, I., Mielnik, B., Plebański, J.: Explicit solution of the continuous Baker-CampbellHausdorff problem. Ann. Phys. 51, 187-200 (1969)

3. Blessenohl, D., Schocker, M.: Noncommutative Character Theory of the Symmetric Group. Imperial College Press (2005)

4. Foissy, L.: Bidendriform bialgebras, trees, and free quasi-symmetric functions. arXiv:math 0505207v1[math.RA]

5. Gelfand, L.M., Krob, D., Lascoux, A., Leclerc, B., Retakh, V., Thibon, J.-Y.: Noncommutative symmetric functions. Adv. Math. 112(2), 218-348 (1995)

6. Magnus, W.: Über Gruppen und zugeordnete Liesche Ringe. J. Reine Angew. Math. 182, 142-149 (1940)

7. Malvenuto, C., Reutenauer, C.: Duality between quasi-symmetric functions and the Solomon descent algebra. J. Algebra 177, 967-982 (1995) 
8. Mielnik, B., Plebański, J.: Combinatorial approach to Baker-Campbell-Hausdorff exponents. Ann. Inst. H. Poincaré Sect. A (N.S.) 12, 215-254 (1970)

9. Patras, F., Reutenauer, C.: Lie representations and an algebra containing Solomon's. J. Algebr. Comb. 16, 301-314 (2002)

10. Reutenauer, C.: Theorem of Poincaré-Birkhoff-Witt, logarithm and representations of the symmetric group whose order are the Stirling numbers. In: Labelle, G., Leroux, P. (eds.) Combinatoire Énumérative. Lecture Notes in Mathematics, vol. 1234, pp. 267-284. Springer, Berlin (1985)

11. Reutenauer, C.: Free Lie Algebras. London Mathematical Society Monographs, vol. 7. Oxford University Press, Oxford (1993). New series

12. Solomon, L.: On the Poincaré-Birkhoff-Witt theorem. J. Comb. Theory (A) 4, 363-375 (1968)

13. Solomon, L.: A Mackey formula in the group ring of a Coxeter group. J. Algebra 41, 255-268 (1967)

14. Sweedler, M.: Hopf Algebras. Benjamin, New York (1969). 